\title{
Agentification of Individuals: A Multi-Agent Approach to Metaheuristics Mehmet Emin Aydin*
}

University of Bedfordshire, Luton, UK

Collective computational intelligence is one of the best ways in problem solving and optimisation since it allows putting collective effort together from a verity of computational methods and well harmonise it. Optimisation problems are very well known hard engineering problems, which have been challenged with various approaches since ever. A category of problem solving approaches for optimisation is known as metaheuristics with which particular problem solving frameworks are implemented with domain information and used to attempt problem solving intelligently. Optimisation is naturally an iterative process in which a search algorithm is devised to look for a feasible solution that provides the best benefit with respect to performance criterion. Any global or local search algorithm designed for optimisation starts with an initial solution and iteratively looks for the best solution with the devised search procedure. Among the approaches, heuristic-based search algorithms are classified as individual-based methods and population-based methods. Collective intelligence is achieved with the techniques including population-based problem solving approaches.

Population-based problem solving methods are devised with a population of individual solutions and a core algorithmic mechanism in which the collective behaviour of a designed algorithm is created and maintained with processing individual solutions included in the population. The core algorithm is mainly independent of the population, where individuals contained in the populations remain inactive and have a role played as the source of static information to be processed. Therefore, the collective behaviour is mainly maintained with the core algorithm which operates upon the individuals, where the contribution of the individuals remains minimal. It is believed that the contribution of individuals towards collaborative behaviour/ intelligence can be improved with furnishing each individual with proactiveness, where individuals can actively collaborate alongside information provision. The main problem here is how to extend the individuals with functionalities to contribute more in optimisation process. Once this is attempted, the requirement for computational power will significantly rise, and more work-load will be queued for processing. There will also be complexity issues in modelling the problems via such extended algorithms, which clearly makes the situation more challenging and justifies attempts to find some way outs.

Multi agent systems and swarm intelligence are two prominent approaches to achieve high performance and quality in problem solving. The concept of multi agent systems (MAS) is one of wellknown and reasonably mature collective intelligence approaches with which a set of proactive agents act individually for solving the problems collaboratively [1]. The idea is to build up teams of intelligent autonomous entities solving the problems in harmony and composing a certain way and level of coordination to let each individual proactively and efficiently collaborate in solving the problems using individual intelligence [2]. The main properties of MAS (i.e. autonomy, responsiveness, redundancy, and distributed approach) facilitate success in MAS applications, which result in a good record in implementations within many research fields including production planning, scheduling and control [3], engineering design, and process planning [1].

Multi agent systems can be used for enhancing population-based search algorithms in the way that the individuals taking part of the search process are allowed to play more proactive role. For this purpose, the agents, which are more specialised with various functionalities, are teamed together such that the skills and functionalities lined up are complementary. The concept of metaheuristic agents is identified to describe such multi agent systems devised with metaheuristics to tackle hard optimisation problems. As mentioned earlier, the main idea is to build up intelligent autonomous agents whose constitute teams to solve the problems in harmony. The agents equipped with metaheuristics aim to solve hard and large-scale problems with their own intelligent search skills. However, it is very costly to furnish all functionalities required into one agent only. Therefore, the functionalities and skills are distributed among the agents and need to be organised and coordinated in a complementary fashion. In the literature, the metaheuristics have been implemented, by large, as standalone applications ordinarily and examined under such circumstances. Few multi agent systems implementing metaheuristics are introduced and overviewed with respect to their performances in the literature by Aydin and Hammami and Ghediera $[4,5]$.

Due to the fact that standalone heuristic search algorithms usually face with local minima problem, various ideas including memetic algorithms, hybrid algorithms etc. have challenged for overcoming such discomforts in search. These approaches solve the problem to some extent, but, not completely. Another idea to tackle this issue would be the usage of multi-agent systems in the way that a well-tailored team of agents can collaboratively approach the problem and solve it seamlessly [6-8]. However, the main challenge, in this respect, is how to organise the agents so that a collective and desirable behaviour would emerge towards solving the problems. In fact, the performances of multiagent systems including metaheuristic teams significantly depend on the quality of collaboration [4]. The harmony achieved in populationbased problem solving algorithms such as evolutionary algorithms, swarm intelligence etc, shows a clear potential to be borrowed for this purpose.

Swarm intelligence is a particular type of collective intelligence, where an intelligent behaviour can emerge as the outcome of a collection of self-organised simple agents, organisms or individuals. Simple organisms that live in colonies; such as ants, bees, bird flocks etc., have long fascinated many people for their collective intelligence that is manifested in many of the things that they do. A population of

*Corresponding author: Mehmet Emin Aydin, University of Bedfordshire, Luton, UK, E-mail: mehmet.aydin@beds.ac.uk

Received September 20, 2013; Accepted September 27, 2013; Published September 30, 2013

Citation: Aydin ME (2013) Agentification of Individuals: A Multi-Agent Approach to Metaheuristics. J Comput Sci Syst Biol 6: e105. doi:10.4172/jcsb.1000e105

Copyright: (c) 2013 Aydin ME. This is an open-access article distributed under the terms of the Creative Commons Attribution License,which permits unrestricted use, distribution, and reproduction in any medium, provided the original author and source are credited. 
Citation: Aydin ME (2013) Agentification of Individuals: A Multi-Agent Approach to Metaheuristics. J Comput Sci Syst Biol 6: e105. doi:10.4172/ jcsb.1000e105

Page 2 of 2

simple units can interact with each other as well as their environment without using any set of external instructions to proceed, and compose a swarm intelligence system. The swarm intelligence approaches produce the collective behaviours of social insects in the way that simple contributions by each individual intelligently form up collective behaviours to tackle complicated issues. This idea sparks for creating ways artificially so that all simple contributions by individuals are counted towards more complicated and collective behaviours. More complicated problems are solved with such swarm intelligence algorithms imitating the behaviour of social insects. Various hard optimisation problems have been solved with a variety of swarm intelligence algorithms [9-11].

As discussed above, the original idea of swarm intelligence is to form up populations of enabled individuals for collaborative problem solving. However, due to computational complexity and the hardship in furnishing the enabled individuals with multiple advanced functionalities, swarms are usually designed as population of individual static solutions evolved with various genetic and/or heuristic operators/ algorithms. Aydin [2] suggests use of swarm intelligence algorithms for the purpose of building collaboration within the teams of intelligent agents. This imposes that the individuals form up the swarms are agentified with various simple and/or advance functionalities such as problem solving and communicating independently. The idea is cultivated as follows: a population of agents is created and developed with search skills operating with simulated annealing algorithm. Then, the population is organised to team up a swarm to solve the problems using their search functionalities alongside interaction abilities. Although there are bits and pieces done with this respect, the idea needs to be further investigated using various other swarm intelligence algorithms to coordinate different homogenous and heterogeneous search agent teams.

\section{References}

1. Ayhan MB, Aydin ME, Oztemel E (2013) A multi-agent based approach for change management in manufacturing enterprises. Journal of Intelligent Manufacturing

2. Aydin ME (2012) Coordinating Metaheuristic agents with team intelligence Journal of Intelligent Manufacturing 23: 991-999.

3. Mohebbi S, Shafaei R (2012) E-Supply network coordination: The design of intelligent agents for buyer-supplier dynamic negotiations. J Intell Manuf 23 : 375-391.

4. Aydin ME (2007) Meta-heuristic agent teams for job shop scheduling problems. LNAI 4659:185-194.

5. Hammami M, Ghediera K (2005) COSATS, X-COSATS: Two multi-agen systems cooperating simulated annealing, tabu search and X-over operator for the K-Graph Partitioning problem. Lecture Notes in Computer Science 3684: 647-653.

6. Kolp M, Giorgini P, Mylopoulos J (2006) Multi-agent architectures as organizational structures. Autonomous Agents and Multi-Agent Systems 13: 3-25.

7. Panait L, Luke S (2005) Cooperative multi-agent learning: The state of the art. Autonomous Agents and Multi-Agent Systems 11: 387-434.

8. Vazquez-Salceda J, Dignum V, Dignum, F (2005) Organizing multi-agent systems. Autonomous Agents and Multi-Agent Systems 11: 307-360.

9. Colorni A, Dorigo M, Maniezzo V, Trubian M (1994) Ant System for Job-Shop Scheduling. Belgian Journal of Operations Research Statistics and Computer Science (JORBEL) 34: 39-53.

10. Kennedy J, Eberhart RC (1995) Particle swarm optimization. Proceedings of IEEE International v Conference on Neural Networks, Piscataway, NJ. pp. 1942-1948.

11. Tasgetiren MF, Liang YC, Sevkli M, Gencyilmaz G (2007) Particle Swarm Optimization Algorithm for Makespan and Total Flowtime Minimization in Permutation Flowshop Sequencing Problem. European Journal of Operational Research 177: 1930-1947. 